\title{
AVALIAÇÃO DA UTILIZAÇÃO DOS ECOPONTOS NOS BAIRROS: FÁTIMA E ESPERANÇA
}

Sâmia de Sousa Rocha - email: sami.sousa1@ hotmail.com Instituto Federal de Educação, Ciência e Tecnologia do Ceará - IFCE Karina Bandeira Cantarini - email: karinacantarini@gmail.com Instituto Federal de Educação, Ciência e Tecnologia do Ceará - IFCE 


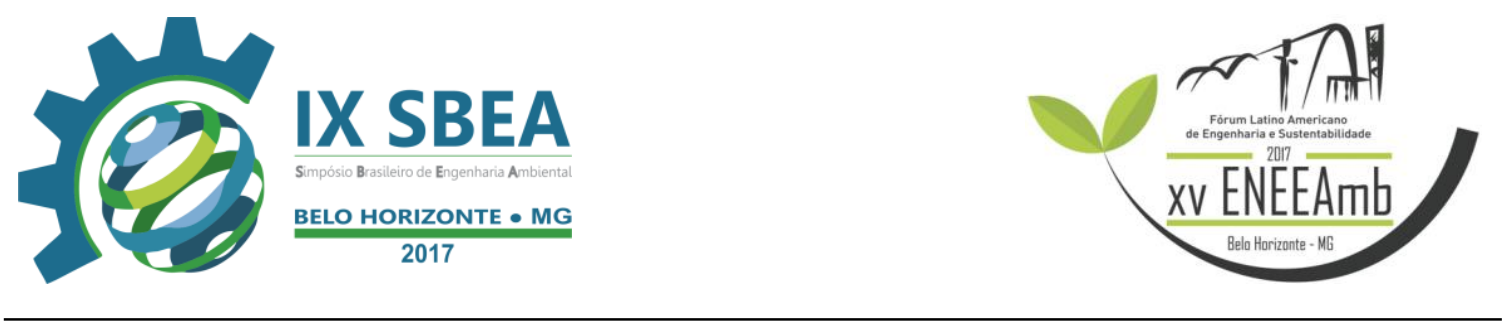

\section{RESUMO}

Tendo em vista os impactos ambientais, sociais e econômicos causados pelo descarte incorreto dos resíduos sólidos na cidade de Fortaleza/Ce, surgiu o projeto de criação dos ecopontos que são postos de descarte de resíduos sólidos que tem como intuito amenizar estes impactos e proporcionar um descarte correto de diversos materiais recicláveis. Com isso, foi realizado um estudo visando informar como ocorreu o processo de instalação deste projeto, quais os materiais que podem ser recebidos nos postos, como se deu a divulgação e o incentivo ao uso dos postos de coleta e também, foi realizado pesquisas em dois ecopontos específicos instalados na capital em diferentes regiões, um localizado no bairro Conjunto Esperança e o outro no bairro de Fátima, obtendo-se com os resultados a caracterização de como se dá a utilização dos ecopontos pela população, quais resíduos são comumente mais descartados e também a viabilidade e as mudanças promovidas através da implantação do projeto ecoponto.

Palavras-chave: Resíduos Sólidos, Reciclagem, Ecopontos.

\section{INTRODUÇÃO/OBJETIVO}

Analisando apenas os RSU (resíduos sólidos urbanos) que engloba os resíduos domiciliares e os de limpeza urbana foi visto que de acordo com a ABRELPE (Associação Brasileira de Empresas de Limpeza Pública e Resíduos Especiais) a geração total de RSU no Brasil em 2014 foi de aproximadamente 78,6 milhões de toneladas, tendo em vista que este é um dos dados disponíveis mais recente até o ano de 2016, sabe-se que esta quantidade de RSU deve ter tido um crescimento constante nos anos posteriores à 2014.

Além disso, de acordo com a ABRELPE é gerado, no estado do Ceará, cerca de 9.700 toneladas de RSU por dia, na qual deste valor é coletado cerca de 7.500 toneladas. Entretanto, desta quantidade coletada apenas cerca de 3.400 toneladas tem uma destinação adequada, a outra parte é destinada aos aterros controlados e aos lixões espalhados pelo estado.

Na cidade de Fortaleza de acordo com a ACFOR (Autarquia de regulamentação, fiscalização e controle dos serviços públicos de saneamento ambiental), desde o mês de janeiro à agosto de 2016 foram coletadas cerca de 319.909,87 toneladas de resíduos 


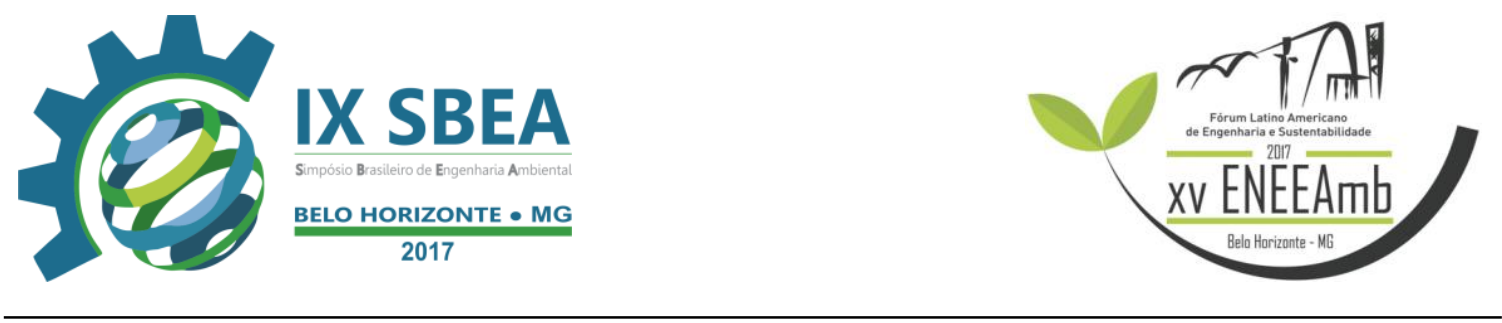

sólidos nos chamados "pontos de lixo" que são locais descarte incorreto de resíduos, 394.491,41 toneladas de lixo domiciliar que contém de forma geral diversos materiais que poderiam ser reciclados e 47.500 toneladas da "coleta seletiva ECOFOR" na qual corresponde a menor quantidade, mostrando assim, a carência da realização da coleta seletiva na capital.

E em meio a essa problemática surgiu o projeto de criação de ecopontos que tem como finalidade a instalação de postos onde é feito a coleta de materiais recicláveis e a sua devida destinação, tendo em vista o grande problema gerado pelo descarte incorreto de resíduos sólidos, que varia desde alagamentos até a proliferação de graves doenças e epidemias.

Dessa forma, a produção desse artigo tem como objetivo principal avaliar a utilização de dois ecopontos o do Esperança e o de Fátima, localizados em diferentes bairros da cidade de Fortaleza, a fim de analisar como a população utiliza os ecopontos e mostrando a sua importância no âmbito social, ambiental e econômico levando em consideração as diferenças sociais e econômicas de cada bairro. Por fim, visando também aferir a quantidade de resíduos recebidos nos postos e as mudanças promovidas por sua instalação.

\section{METODOLOGIA}

A obtenção dos dados ocorreu a partir de questionários e entrevistas realizadas com a população no entorno dos ecopontos entre os dias 19 e 29 de agosto de 2016) E também, com uma entrevista realizada na Secretaria de Conservação e Serviços Públicos (SCSP) com os responsáveis pela implantação do projeto ecoponto na capital.

\section{RESULTADOS E DISCUSSÃO}

1. Implantação do projeto ecoponto.

O projeto teve seu primeiro posto de coleta inaugurado em novembro de 2015 no bairro de Fátima. Após esta inauguração foi dado início a uma série de outras implantações em diversos bairros, sendo estes escolhidos após uma análise do bairro onde primordialmente era visto se o bairro possuía uma área pública disponível e adequada para receber o posto, sendo de preferência um espaço "abandonado", assim 


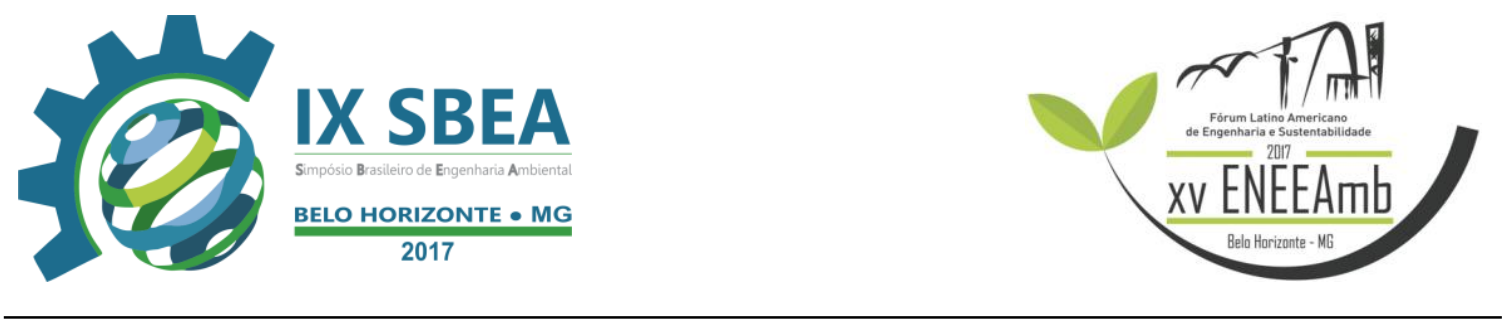

transformando e revitalizando uma área degradada. O projeto prevê ao decorrer dos próximos 4 anos conseguir realizar a instalação de um ecoponto em cada bairro da cidade de Fortaleza.

2. Resíduos sólidos que podem ser descartados nos ecopontos

Os postos recebem desde materiais mais conhecidos como o papel, plástico, vidro e metal à pequenas proporções de entulhos, restos de poda, baterias, embalagens do tipo tetra pak, móveis e estofados velhos, pneus velhos e até mesmo óleo de cozinha, todos esses materiais são destinados a empresas de reciclagens credenciadas que realizam a devida destinação dos resíduos.

\section{Parcerias institucionais}

Com o intuito de incentivar o descarte dos seus resíduos nos ecopontos foi criado o projeto "Recicla Fortaleza" que proporcionou diversas parcerias A primeira ocorreu em maio de 2016 entre a Prefeitura de Fortaleza e a Companhia Energética do Ceará (Coelce), na qual foi disponibilizado descontos na conta de energia em troca do resíduo descartado. A segunda parceria foi com a Empresa de Transporte Urbano de Fortaleza (Etufor), que possibilitou crédito no bilhete de transporte urbano da capital em troca do descarte no ecoponto. Além disso, tem encaminhada outra parceria que será com a Companhia de Água e Esgoto do Ceará (Cagece).

Todas as parcerias funcionam da mesma forma: os resíduos descartados pelos cidadãos são pesados e de acordo com uma tabela de preço o peso do material é reinvestido em reais/créditos, isso tudo através de um cadastrado feito no próprio ecoponto. A implementação do projeto Recicla Fortaleza já mostrou ter resultados positivos, pois desde sua implantação foi crescente o aumento no número de descartes realizados e além disso, também colabora na divulgação dos postos de coleta.

\section{Utilização do ecoponto Esperança}

Com o questionário realizando no entorno do ecoponto Esperança, foi primeiramente, analisado que dos entrevistados $90 \%$ são moradores do bairro e os outros $10 \%$ são moradores de bairros próximos desprovidos de um ecoponto e com isso 


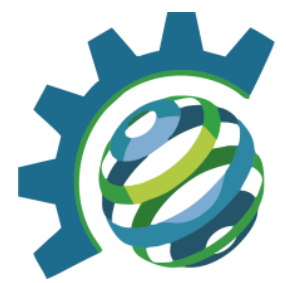

se deslocam para o bairro Conjunto Esperança, afim de utilizar o posto de coleta, este e outros dados podem ser analisados na Figura 1.

Figura 1 - Resultado das entrevistas no ecoponto Esperança em Fortaleza (2016)

\begin{tabular}{|c|c|c|c|c|c|c|c|c|c|}
\hline \multicolumn{10}{|c|}{ ECOPONTO: ESPERANÇA } \\
\hline \multicolumn{10}{|c|}{ QUANTIDADE DE PESSOAS ENTREVISTADAS: 20} \\
\hline \multirow{3}{*}{$\begin{array}{l}\text { PESQUISA REALIZADA } \\
\text { NO POSTO DE COLETA? }\end{array}$} & & SIM & NÃO & \multirow{3}{*}{\multicolumn{2}{|c|}{$\begin{array}{c}\text { SEXO DO } \\
\text { ENTREVISTADO (A): }\end{array}$}} & & MASCULINO & \multicolumn{2}{|c|}{ FEMININO } \\
\hline & Q & 16 & 4 & & & $Q$ & 13 & \multicolumn{2}{|c|}{7} \\
\hline & $\%$ & $80 \%$ & $20 \%$ & & & $\%$ & $65 \%$ & \multicolumn{2}{|c|}{$35 \%$} \\
\hline \multirow{3}{*}{$\begin{array}{l}\text { MORADOR(A) DO } \\
\text { BAIRRO? }\end{array}$} & & SIM & NÃO & \multirow{3}{*}{\multicolumn{2}{|c|}{ UTILIZA O ECOPONTO? }} & & SIM & \multicolumn{2}{|c|}{ NÅO } \\
\hline & Q & 18 & 2 & & & $Q$ & 19 & \multicolumn{2}{|c|}{1} \\
\hline & $\%$ & $90 \%$ & $10 \%$ & & & $\%$ & $95 \%$ & \multicolumn{2}{|c|}{$5 \%$} \\
\hline \multirow{3}{*}{ FAIXA ETÁRIA: } & & $\begin{array}{c}\text { ENTRE } 15 \mathrm{E} \\
20\end{array}$ & $\begin{array}{c}\text { ENTRE 21 E } \\
25\end{array}$ & $\begin{array}{c}\text { ENTRE } 26 \mathrm{E} \\
30\end{array}$ & \begin{tabular}{|c|} 
ENTRE $31 \mathrm{E}$ \\
35
\end{tabular} & $\begin{array}{c}\text { ENTRE } 36 \mathrm{E} \\
40\end{array}$ & ENTRE 41 E 45 & \begin{tabular}{|c|} 
ENTRE $46 \mathrm{E}$ \\
50
\end{tabular} & $\begin{array}{c}\text { ACIMA DE } \\
50\end{array}$ \\
\hline & $Q$ & 0 & 2 & 1 & 1 & 1 & 3 & 5 & 7 \\
\hline & $\%$ & $0 \%$ & $10 \%$ & $5 \%$ & $5 \%$ & $5 \%$ & $15 \%$ & $25 \%$ & $35 \%$ \\
\hline \multirow{3}{*}{$\begin{array}{l}\text { POR QUAL MEIO TEVE } \\
\text { CIÊNCIA DO } \\
\text { ECOPONTO? }\end{array}$} & & INTERNET & TV & \multicolumn{2}{|c|}{ AMIGOS/FAMILIARES } & $\begin{array}{l}\text { INAUGURA } \\
\text { ÇÃOO }\end{array}$ & \multicolumn{2}{|c|}{ NA CONSTRUÇÃO } & OUTROS \\
\hline & $Q$ & 0 & 1 & 7 & 7 & 4 & 6 & & 2 \\
\hline & $\%$ & $0 \%$ & $5 \%$ & 35 & & $20 \%$ & $30 \%$ & & $10 \%$ \\
\hline \multirow{3}{*}{$\begin{array}{l}\text { FREQUÊNCIA DA IDA AO } \\
\text { ECOPONTO: }\end{array}$} & & \multicolumn{2}{|c|}{ PRIMEIRA VEZ } & \multicolumn{2}{|c|}{ A CADA 7 DIAS } & $\begin{array}{c}\text { A CADA } 15 \\
\text { DIAS }\end{array}$ & \multicolumn{2}{|c|}{ A CADA 30 DIAS } & OUTROS \\
\hline & $Q$ & \multicolumn{2}{|c|}{3} & \multicolumn{2}{|c|}{3} & 1 & \multicolumn{2}{|l|}{7} & 4 \\
\hline & $\%$ & \multicolumn{2}{|c|}{$15 \%$} & \multicolumn{2}{|c|}{$15 \%$} & $5 \%$ & \multicolumn{2}{|c|}{$35 \%$} & $20 \%$ \\
\hline \multirow{3}{*}{$\begin{array}{c}\text { RESÍDUOS MAIS } \\
\text { DESCARTADO PELO } \\
\text { ENTREVISTADO (A): }\end{array}$} & & PAPEL & PLÁSTICO & VIDRO & METAL & ENTULHO & VOLUMOSOS & OUTROS & TODOS \\
\hline & $Q$ & 3 & 6 & 3 & 3 & 1 & 2 & 4 & 3 \\
\hline & $\%$ & $12 \%$ & $24 \%$ & $12 \%$ & $12 \%$ & $4 \%$ & $8 \%$ & $16 \%$ & $12 \%$ \\
\hline \multirow{3}{*}{$\begin{array}{l}\text { PRETENDE CONTINUAR } \\
\text { DESCARTANTO? }\end{array}$} & & SIM & NÃO & \multirow{3}{*}{\multicolumn{2}{|c|}{$\begin{array}{l}\text { VOCÊ INCENTIVA A } \\
\text { UTILIZAÇÃ DOS } \\
\text { ECOPONTOS? }\end{array}$}} & & SIM & \multicolumn{2}{|c|}{ NÃO } \\
\hline & $Q$ & 19 & 1 & & & $Q$ & 20 & \multicolumn{2}{|c|}{0} \\
\hline & $\%$ & $95 \%$ & $5 \%$ & & & $\%$ & $100 \%$ & \multicolumn{2}{|c|}{$0 \%$} \\
\hline
\end{tabular}

Levando em consideração que $95 \%$ dos entrevistados afirmaram que utilizam o ecoponto, foi visto que o número de homens acima de 50 anos que utilizam o posto de coleta é elevado, como pode ser observado na figura 2. Isso provavelmente deve-se ao fato que o estabelecimento funciona em horário comercial, logo a maioria das pessoas mais jovens estão exercendo alguma atividade.

Figura 2 - Relação entre o sexo e faixa etária dos entrevistados - Fortaleza (2016)

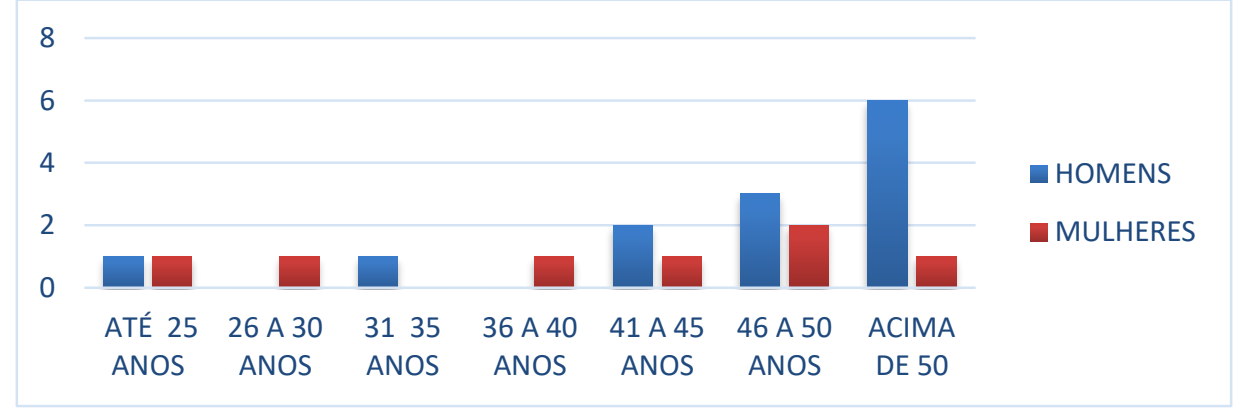




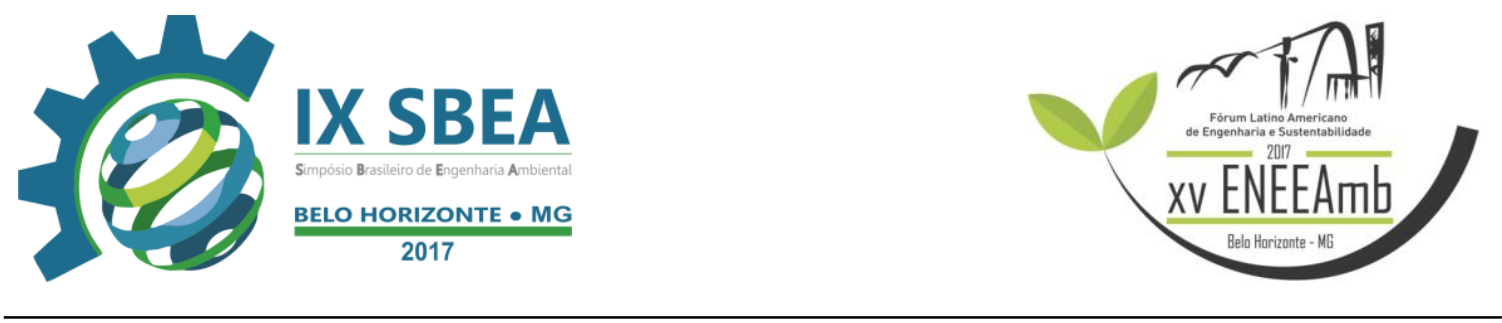

No quesito da forma em que os entrevistados tiveram ciência da existência do ecoponto, a maioria informou que foi por amigos, familiares e/ou vizinhos, outra grande parte informou que acompanhou a construção dos postos, porém não tinham ciência que seria postos de coleta de resíduos.

Isso mostra a carência de uma divulgação mais efetiva através dos meios de comunicação e também de outras formas de divulgação, como em escolas e locais públicos. Pois, o bairro Conjunto Esperança tem de cerca de 16.405 habitantes, contudo, a grande maioria dos moradores do bairro não tem conhecimento da existência de um ecoponto e dos poucos moradores que tem ciência do posto de coleta grande parte não sabe dos benefícios econômicos que se pode ter ao realizar o descartar seus resíduos.

Foi visto também que, $35 \%$ dos entrevistados informaram que realizam o descarte a cada trinta dias, pois esperam acumular uma quantidade significativa de resíduos para então realizar o descarte. As porcentagens a respeito dos resíduos mais descartados foram visivelmente niveladas como mostrado na Figura 3.

Figura 3 - Gráfico do índice de resíduos descartados - Fortaleza (2016).

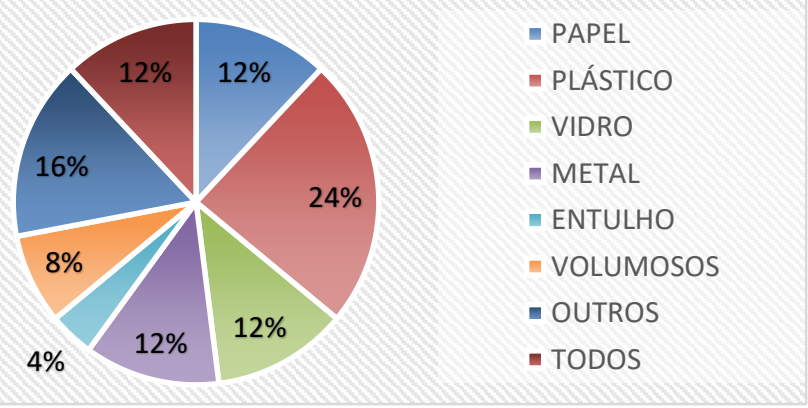

O material mais descartado é o plástico, provavelmente, por ser o material mais abundante em embalagens e presente em diversos produtos, em seguida é o papel. Os 16\% que corresponde aos resíduos classificados como "outros" são materiais como: eletroeletrônicos, baterias, podas entre outros que em baixa quantidade são descartados.

Ao perguntar aos entrevistados o motivo pelo qual o ecoponto é importante, obtivemos diversas respostas como, "Para manter a cidade limpa, trazer melhoria de vida para população e por conta do desconto na conta de luz", resposta de uma senhora com idade acima dos 50 anos. 


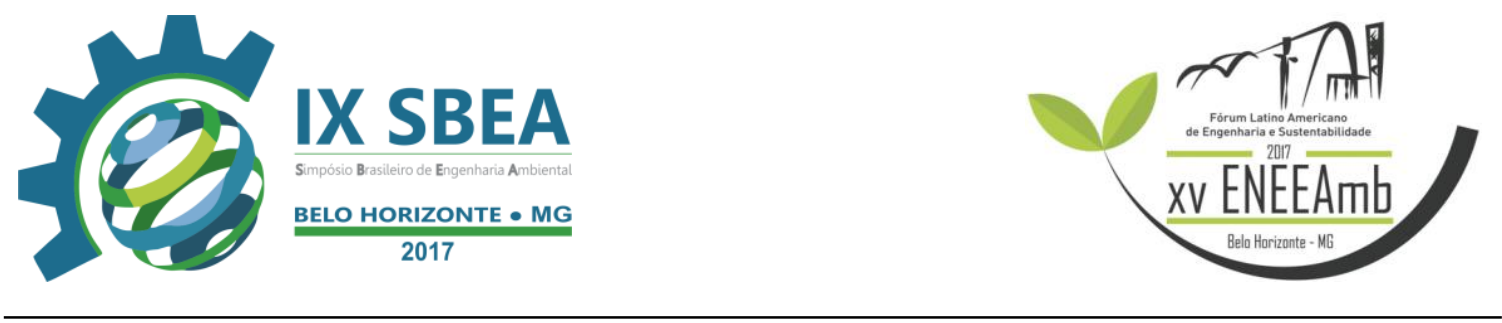

Isso nos mostrou que grande parte tem ciência da importância social e ambiental da instalação dos ecopontos, porém, que o incentivo é parte crucial do total compromisso que os moradores têm em descartar os resíduos no ecoponto.

Apesar da instalação do ecoponto ter sido em um local antes muito degradado, pois havia um "ponto de lixo" ainda é observado nas redondezas outros "pontos de lixo", portanto nota-se que a grande maioria da população ainda não tem a devida educação ambiental o que gera revolta nos moradores que utilizam o ecoponto e possuem mais consciência dos impactos que esses resíduos pode causar na cidade, que além de gerar alagamentos, também é um grande causador de doenças, que podem levar até a morte.

De acordo com o IBGE o Índice de Desenvolvimento humano (IDH) do bairro Esperança é de cerca de 0,2879 sendo considerado baixo e estando na $76^{\circ}$ posição no ranking dos índices de IDH de todos os bairros da capital e como o IDH é uma medida composta de indicadores de saúde, educação e renda, isso mostra que o bairro tem uma carência em educação e que isso influência diretamente na questão social e ambiental.

\section{Utilização do ecoponto Fátima}

Como resultado das entrevistas realizadas no ecoponto do bairro de Fátima para obter os dados a respeito da sua utilização, foi observado primeiramente, que apenas 16 entrevistados utilizam o ecoponto os outros quatro entrevistados afirmaram não utilizar e também não ter conhecimento de como funciona o posto de coleta, como mostrado na Figura 4.

Também foi visto que apenas $55 \%$ dos entrevistados moram no bairro (salientando que os 4 entrevistados que não utilizam o ecoponto moram no bairro), este resultado pode estar relacionado ao fato de que o bairro de Fátima é de modo geral composto por prédios comerciais, diversos estabelecimento e geograficamente está localizado próximo à bairros que são residenciais e que não possuem ecopontos e com isso diversas pessoas se desloca para o bairro de Fátima, afim de utilizar o ecoponto. 
Figura 4 - Resultado das entrevistas no ecoponto Fátima em Fortaleza (2016)

\begin{tabular}{|c|c|c|c|c|c|c|c|c|c|}
\hline \multicolumn{10}{|c|}{ ECOPONTO: FÁTIMA } \\
\hline \multicolumn{10}{|c|}{ QUANTIDADE DE PESSOAS ENTREVISTADAS: 20} \\
\hline \multirow{3}{*}{$\begin{array}{l}\text { PESQUISA REALIZADA } \\
\text { NO POSTO DE COLETA? }\end{array}$} & & SIM & NÃO & \multirow{3}{*}{\multicolumn{2}{|c|}{$\begin{array}{c}\text { SEXO DO } \\
\text { ENTREVISTADO (A): }\end{array}$}} & & MASCULINO & \multicolumn{2}{|c|}{ FEIMININO } \\
\hline & Q & 16 & 4 & & & $Q$ & 9 & \multicolumn{2}{|c|}{11} \\
\hline & $\%$ & $80 \%$ & $20 \%$ & & & $\%$ & $45 \%$ & \multicolumn{2}{|c|}{$55 \%$} \\
\hline \multirow{3}{*}{$\begin{array}{l}\text { MORADOR(A) DO } \\
\text { BAIRRO? }\end{array}$} & & SIM & NÃO & \multirow{3}{*}{\multicolumn{2}{|c|}{ UTILIZA O ECOPONTO? }} & & SIM & \multicolumn{2}{|c|}{ NÂO } \\
\hline & $Q$ & 11 & 9 & & & $Q$ & 16 & \multicolumn{2}{|c|}{4} \\
\hline & $\%$ & $55 \%$ & $45 \%$ & & & $\%$ & $80 \%$ & \multicolumn{2}{|c|}{$20 \%$} \\
\hline \multirow{3}{*}{ FAIXA ETÁRIA: } & & \begin{tabular}{|c|} 
ENTRE $15 \mathrm{E}$ \\
20 \\
\end{tabular} & \begin{tabular}{|c|} 
ENTRE $21 \mathrm{E}$ \\
25 \\
\end{tabular} & \begin{tabular}{|c|} 
ENTRE $26 \mathrm{E}$ \\
30 \\
\end{tabular} & \begin{tabular}{|c|} 
ENTRE $31 \mathrm{E}$ \\
35 \\
\end{tabular} & \begin{tabular}{|c|} 
ENTRE $36 \mathrm{E}$ \\
40 \\
\end{tabular} & ENTRE $41 \mathrm{E} 45$ & \begin{tabular}{|c|} 
ENTRE $46 \mathrm{E}$ \\
50 \\
\end{tabular} & $\begin{array}{c}\text { ACIMA DE } \\
50 \\
\end{array}$ \\
\hline & Q & 4 & 1 & 2 & 2 & 3 & 1 & 1 & 6 \\
\hline & $\%$ & $20 \%$ & $5 \%$ & $10 \%$ & $10 \%$ & $15 \%$ & $5 \%$ & $5 \%$ & $30 \%$ \\
\hline \multirow{3}{*}{$\begin{array}{l}\text { POR QUAL MEIO TEVE } \\
\text { CIÊNCIA DO } \\
\text { ECOPONTO? }\end{array}$} & & INTERNET & TV & \multicolumn{2}{|c|}{ AMIGOS/FAMILIARES } & \begin{tabular}{|c|} 
CONSTRUÇ \\
$\tilde{\text { ÃO }}$ \\
\end{tabular} & \multicolumn{2}{|c|}{ PASSANDO EM FRENTE } & OUTROS \\
\hline & $Q$ & 2 & 0 & 3 & 3 & 1 & 14 & & 0 \\
\hline & $\%$ & $10 \%$ & $0 \%$ & 15 & & $5 \%$ & $70 \%$ & & $0 \%$ \\
\hline \multirow{3}{*}{$\begin{array}{l}\text { FREQUÊNCIA DA IDA AO } \\
\text { ECOPONTO: }\end{array}$} & & \multicolumn{2}{|c|}{ PRIMEIRA VEZ } & \multicolumn{2}{|c|}{ A CADA 7 DIAS } & $\begin{array}{c}\text { A CADA } 15 \\
\text { DIAS }\end{array}$ & A CADA 3 & 0 DIAS & NÃO USA \\
\hline & $Q$ & \multicolumn{2}{|c|}{3} & \multicolumn{2}{|c|}{1} & 4 & \multicolumn{2}{|l|}{8} & 4 \\
\hline & $\%$ & \multicolumn{2}{|c|}{$15 \%$} & \multicolumn{2}{|c|}{$5 \%$} & $20 \%$ & \multicolumn{2}{|c|}{$40 \%$} & $20 \%$ \\
\hline \multirow{3}{*}{$\begin{array}{c}\text { RESÍDUOS MAIS } \\
\text { DESCARTADO PELO } \\
\text { ENTREVISTADO (A): }\end{array}$} & & PAPEL & PLÁSTICO & VIDRO & METAL & ENTULHO & VOLUMOSOS & TETRA PAK & OUTROS \\
\hline & $Q$ & 7 & 11 & 5 & 0 & 1 & 2 & 6 & 1 \\
\hline & $\%$ & $21,21 \%$ & $33,33 \%$ & $15,15 \%$ & $0,00 \%$ & $3,03 \%$ & $6,06 \%$ & $18,18 \%$ & $3,03 \%$ \\
\hline \multirow{3}{*}{$\begin{array}{l}\text { PRETENDE CONTINUAR } \\
\text { DESCARTANTO? }\end{array}$} & & SIM & NÃO & \multirow{3}{*}{\multicolumn{2}{|c|}{$\begin{array}{l}\text { VOCÊ INCENTIVA A } \\
\text { UTILIZAÇÃ DOS } \\
\text { ECOPONTOS? } \\
\end{array}$}} & & SIM & \multicolumn{2}{|c|}{ NÂO } \\
\hline & $Q$ & 16 & 4 & & & $Q$ & 17 & \multicolumn{2}{|c|}{3} \\
\hline & $\%$ & $80 \%$ & $20 \%$ & & & $\%$ & $85 \%$ & \multicolumn{2}{|c|}{$15 \%$} \\
\hline
\end{tabular}

Além disso, 35\% das pessoas, destinam seus resíduos a cada 30 dias, pois, esperam os seus resíduos acumular para realizar o descarte, estas pessoas na sua maioria estão na faixa etária de 50 anos. Sendo que, os entrevistados com faixa etária acima dos 50 anos, destinam seus resíduos principalmente com finalidade de receber desconto na conta de luz. Portanto, nota-se novamente a importância das parcerias institucionais.

Outro ponto importante é como os entrevistados souberam da existência dos ecopontos $70 \%$ respondeu que foi passando em frente, apenas $10 \%$ pela internet e $0 \%$ pela TV, ou seja, pode-se deduzir que a divulgação do ecoponto, seja na inauguração ou depois não foi feita de forma satisfatória, especialmente, tendo em vista que, $20 \%$ dos entrevistados estão na faixa etária de entre 15 à 20 anos, ou seja, pessoas que são público alvo da internet.

Ademais, os resíduos domésticos foram os mais citados no quesito "materiais descartados" - lembrando que poderia ser citado mais de uma opção por pessoa.

Podemos destacar também, o IDH do bairro que se encontra próximo a 0,6947 consideravelmente alto e relacionar com o fato de os materiais mais destinados serem os plásticos, em geral, o papel e as embalagens do tipo tetra pak, que são materiais que 


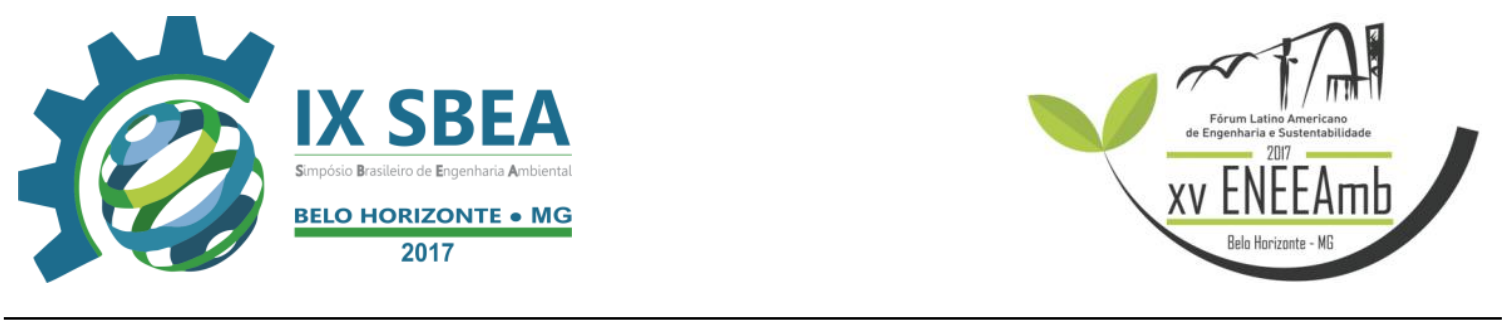

possuem uma decomposição difícil, ou seja, os usuários tem um certo nível de conhecimento ambiental a respeito da reciclagem do material tetra pak, diferentemente do ecoponto do Esperança que nenhuma entrevistado relatou a destinação de embalagens do tipo tetra pak.

Também foi visto que, com exceção das pessoas que não utilizam os ecopontos a maioria dos entrevistados afirmaram que pretendem continuar utilizando, o que é de grande relevância pois significa que as pessoas reconhecem que os ecopontos são bons.

A instalação deste ecoponto também trouxe outros benefícios como a revitalização do local, que antes era mal iluminado e pouco movimentado, fazendo com que o local se tornasse inseguro e à mercê de criminosos, em outros dias o local servia como estacionamento público e ilegal para festas.

\section{CONCLUSÕES}

Dessa forma conclui-se que os ecopontos analisados estão sendo amplamente utilizados pela população independentemente das características do bairro ou IDH do mesmo.

Notou-se que o ecoponto do bairro Esperança é mais utilizado pela comunidade local e o de Fátima é utilizado por moradores locais e de bairros próximos e, portanto, é notório a importância da implantação deste projeto nos outros bairros. Logo, é visto a importância de se dá continuidade aos investimentos em implantações, manutenção e em novos funcionários pois os ecopontos tendem a ser cada vez mais bem-sucedido e com isso podem ganhar visibilidade ao ponto da prefeitura de cidades da RMF (Região metropolitana de Fortaleza) e de outras cidades distantes também aderirem ao projeto e ajudar amenizar o grande impacto dos resíduos sólidos.

Contudo, também se concluiu que falta comprometimento dos órgãos responsáveis em divulgar os postos de coleta e incentivar a população à utiliza-los, pois grande parcela dos moradores ainda não sabem como funciona e qual a importância e os benefícios de se utilizar um ecoponto

Além disso, as instalações dos ecopontos geraram inúmeros benefícios para Fortaleza, impactando sobre problemas sociais, econômicos e acima de tudo, ecológicos. também, proporcionando a reabilitação de locais antes utilizados de forma 


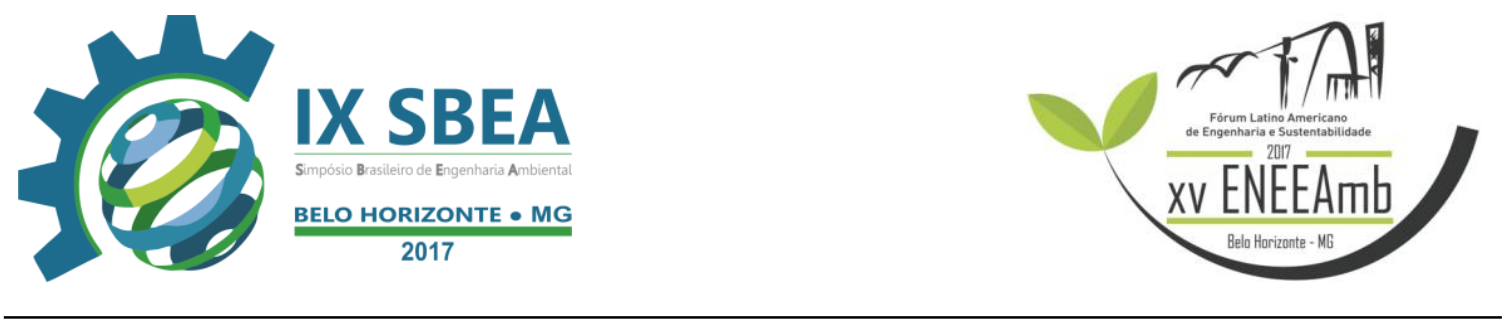

incorreta e contribuindo para a limpeza e segurança da cidade, além de proporcionar o bem-estar da população.

Por fim, apesar dos resultados mostrarem que muitos moradores utilizam os ecopontos é notório a carência da educação ambiental na vida da população, que ainda insiste em realizar descartes incorretos mesmo existindo diversas formas adequadas de se destinar os resíduos sólidos, portanto, é crucial a implementação da educação ambiental nesta e nas próximas gerações.

\section{REFERÊNCIAS BIBLIOGRÁFICAS}

ABRELPE: Panorama de 2014. Disponível em: http://www.abrelpe.org.br/Panorama/panorama2014.pdf. Acesso em: 07 set .2016.

IBGE: Coleta de lixo nas cidades do Brasil. Disponível em: http://www.ibge.gov.br/home/estatistica/populacao/condicaodevida/pnsb/lixo_coletado/ lixo_coletado110.shtm. Acesso em: 28 set. 2016.

IDH: Lista por bairros de Fortaleza. Disponível em: http://moises-deoliveira.blogspot.com.br/2015/04/lista-do-bairro-defortaleza-por-indice.html. Acesso em: 01 set. 2016.

JORNAL ONLINNE OPOVO: Pontos de lixo em Fortaleza. Disponível em: http://www.opovo.com.br/app/opovo/cotidiano/2015/08/19/noticiasjornalcotidiano,3489 653/pontos-de-lixo-em-fortaleza-geram-68-denuncias-por-dia.shtml. Acesso em: 28 set. 2016.

POPULAÇÃO NET: Números de habitantes no bairro Conjunto Esperança. Disponível em: http://populacao.net.br/populacao-conjunto-esperanca_fortaleza_ce.html. Acesso em: 28 set. 2016.

POPULAÇÃO NET: Números de habitantes no bairro de Fátima. Disponível em: http://populacao.net.br/populacao-fatima_fortaleza_ce.htm>. Acesso em: 28 set. 2016. 